\title{
An Optimal Inequality for One-Parameter Mean
}

\author{
Hongya Gao, Yanjie Zhang, Tian Wang \\ College of Mathematics and Computer Science, Hebei University, Baoding, China \\ Email: ghy@hbu.cn,347764565@qq.com,260907818@qq.com
}

Received September 16, 2013; revised October 15, 2013; accepted October 21, 2013

Copyright (C) 2013 Hongya Gao et al. This is an open access article distributed under the Creative Commons Attribution License, which permits unrestricted use, distribution, and reproduction in any medium, provided the original work is properly cited.

\section{ABSTRACT}

In the present paper, we answer the question: for $0<\alpha<1$ fixed, what are the greatest value $p(\alpha)$ and the least value $q(\alpha)$ such that the inequality $J_{p}(a, b)<A^{\alpha}(a, b) G^{1-\alpha}(a, b)<J_{q}(a, b)$ holds for all $a, b>0$ with $a \neq b$ ? where for $p \in R$, the one-parameter mean $J_{p}(a, b)$, arithmetic mean $A(a, b)$ and geometric mean $G(a, b)$ of two positive real numbers $a$ and $b$ are defined by $J_{p}(a, b)=\left\{\begin{array}{ll}a, & a \neq b, \\ \frac{p\left(a^{p+1}-b^{p+1}\right)}{(p+1)\left(a^{p}-b^{p}\right)}, & a \neq b, p \neq-1,0, \\ \frac{a b(\log a-\log b)}{a-b}, & a \neq b, p=-1, \\ \frac{a-b}{\log a-\log b}, & a \neq b, p=0,\end{array} \quad A(a, b)=\frac{a+b}{2}\right.$ and $G(a, b)=\sqrt{a b}$, respectively.

Keywords: Optimal Inequality; One-Parameter Mean; Arithmetic Mean; Geometric Mean

\section{Introduction}

For $p \in R$, the one-parameter mean $J_{p}(a, b)$, arithmetic mean $A(a, b)$ and geometric mean $G(a, b)$ of two positive real numbers $a$ and $b$ are defined by

$$
J_{p}(a, b)= \begin{cases}a, & a \neq b, \\ \frac{p\left(a^{p+1}-b^{p+1}\right)}{(p+1)\left(a^{p}-b^{p}\right)}, & a \neq b, p \neq-1,0, \\ \frac{a b(\log a-\log b)}{a-b}, & a \neq b, p=-1 \\ \frac{a-b}{\log a-\log b}, & a \neq b, p=0\end{cases}
$$

$$
A(a, b)=\frac{a+b}{2} \text { and } G(a, b)=\sqrt{a b} \text {, respectively. }
$$

There has been some literature on the one-parameter mean values $J_{p}(a, b)$, see [1-6]. It is well-known that the one-parameter mean $J_{p}(a, b)$ is continuous and strictly increases with respect to $p \in R$ for fixed $a, b>0$ with $a \neq b$. Many means are special cases of the one-parameter mean, for example:

$$
J_{1}(a, b)=\frac{a+b}{2}=A(a, b) \text {, the arithmetic mean, }
$$

$J_{1 / 2}(a, b)=\frac{a+\sqrt{a b}+b}{3}=H e(a, b)$, the Heronian mean,

$J_{-1 / 2}(a, b)=\sqrt{a b}=G(a, b)$, the geometric mean, and

$J_{-2}(a, b)=\frac{2 a b}{a+b}=H(a, b)$, the harmonic mean.

In [1], Gao and Niu found the greatest values $p, s_{1}$ and the least values $q, s_{2}$ such that the inequalities

$$
J_{p}(a, b) \leq A^{\alpha}(a, b) G^{\beta}(a, b) H^{1-\alpha-\beta}(a, b) \leq J_{q}(a, b)
$$

and

$$
G_{s_{1}, 1}(a, b) \leq A^{\alpha}(a, b) G^{\beta}(a, b) H^{1-\alpha-\beta}(a, b) \leq G_{s_{2}, 1}(a, b)
$$

hold for all $a, b>0$ with $a \neq b$, where $\alpha+\beta \in(0,1)$, 
and $G_{s, 1}(a, b)=\left[\frac{\left(a^{s}+b^{s}\right)}{(a+b)}\right]^{1 /(s-1)}$, as the Gini mean.

In [2], Cheune and Qi proved the logarithmic convexiity of the one-parameter mean values $J_{p}(a, b)$ and presented the monotonicity of $J(-r) J(r)$ for $r \in R$.

In [3], Wang, Qiu and Chu obtained the greatest value $r_{1}$ and the least value $r_{2}$ such that the double inequality

$$
J_{r_{1}}(a, b) \leq \alpha A(a, b)+(1-\alpha) H(a, b) \leq J_{r_{2}}(a, b)
$$

holds for all $a, b>0$ with $a \neq b$.

In [4], $\mathrm{Hu}, \mathrm{Tu}$ and Chu presented the greatest value $r_{1}$ and the least value $r_{2}$ such that the double inequality $J_{r_{1}}(a, b) \leq T(a, b) \leq J_{r_{2}}(a, b)$ holds for all $a, b>0$ with $a \neq b$, where

$$
T(a, b)=\frac{2 a b}{2 \arctan \left(\frac{(a-b)}{(a+b)}\right)}
$$

denotes the first Seiffert mean.

In [5], Long and Chu found the greatest value $p$ and the least value $q$ such that the inequality

$$
J_{p}(a, b) \leq \alpha A(a, b)+(1-\alpha) H(a, b) \leq J_{q}(a, b)
$$

holds for all $a, b>0$ with $a \neq b$.

In [6], the authors established Schur-convexities of two types of one-parameter mean values in $n$ variables, and obtained Schur-convexities of some well-known functions.

The purpose of this paper is to answer the question: for $0<\alpha<1$ fixed, what are the greatest value $p(\alpha)$ and the least value $q(\alpha)$ such that the inequality

$$
J_{p}(a, b)<A^{\alpha}(a, b) G^{1-\alpha}(a, b)<J_{q}(a, b)
$$

holds for all $a, b>0$ with $a \neq b$ ?

\section{A Preliminary Lemma}

In order to prove the main theorem of this paper, we need the following lemma.

Lemma 2.1. For all $t>1$, one has

$$
m(t)=\frac{t(t+1) \log ^{3} t}{2(t-1)^{3}}<1 .
$$

Proof. The logarithmic derivative of $m(t)$ is

$$
\frac{m^{\prime}(t)}{m(t)}=[\log m(t)]^{\prime}=\frac{n(t)}{t\left(t^{2}-1\right) \log t},
$$

where

$$
n(t)=-\left(t^{2}+4 t+1\right) \log t+3\left(t^{2}-1\right), \lim _{t \rightarrow 1^{+}} n(t)=0 .
$$

Simple calculations lead to

$$
\begin{aligned}
& n^{\prime}(t)=5 t-4-\frac{1}{t}-2(t+2) \log t, \lim _{t \rightarrow 1^{+}} n^{\prime}(t)=0 \\
& n^{\prime \prime}(t)=3-\frac{4}{t}+\frac{1}{t^{2}}-2 \log t, \lim _{t \rightarrow 1^{+}} n(t)=0, \\
& n^{\prime \prime \prime}(t)=\frac{-2(t-1)^{2}}{t^{3}}<0 .
\end{aligned}
$$

(2) follows from (3)-(7) and the fact

$$
\lim _{t \rightarrow 1^{+}} m(t)=1 \text {. }
$$

\section{Main Result}

The main result of this paper is the following theorem.

Theorem 3.1. Let $0<\alpha<1$. Then for any $a, b>0$ with $a \neq b$, we have

$$
J_{\frac{\alpha-1}{2}}(a, b)<A^{\alpha}(a, b) G^{1-\alpha}(a, b)<J_{\frac{3 \alpha-1}{2}}(a, b) .
$$

Moreover, the bounds $J_{\frac{\alpha-1}{2}}(a, b)$ and $J_{\frac{3 \alpha-1}{2}}(a, b)$ are optimal.

Proof. It is no loss of generality to assume that $a>b$. Let $t^{2}=\frac{a}{b}>1, \quad p \in\left\{\frac{\alpha-1}{2}, \frac{3 \alpha-1}{2}\right\}$ and

$$
f_{1}(t)=\frac{J_{p}\left(t^{2}, 1\right)}{A^{\alpha}\left(t^{2}, 1\right) G^{1-\alpha}\left(t^{2}, 1\right)},
$$

then

$$
\frac{f_{1}^{\prime}(t)}{f_{1}(t)}=\left[\log f_{1}(t)\right]^{\prime}=\frac{g_{1}(t)}{t\left(t^{2}+1\right)\left(t^{2 p}-1\right)\left(t^{2 p+2}-1\right),}
$$

where

$$
\begin{aligned}
g_{1}(t)= & (1-\alpha) t^{4 p+4}+(\alpha+1) t^{4 p+2}+(\alpha-2 p-1) t^{2 p+4} \\
& +(2 p+1-\alpha) t^{2 p}-(\alpha+1) t^{2}+\alpha-1 \\
= & (1-\alpha) x^{2 p+2}+(\alpha+1) x^{2 p+1}+(\alpha-2 p-1) x^{p+2} \\
& +(2 p+1-\alpha) x^{p}-(\alpha+1) x+\alpha-1 \\
= & h_{1}(x),
\end{aligned}
$$

where $x=t^{2}>1$. Simple calculations lead to

$$
\begin{aligned}
& \lim _{x \rightarrow 1^{+}} h_{1}(x)=0, \\
h_{1}^{\prime}(x)= & 2(p+1)(1-\alpha) x^{2 p+1}+(2 p+1)(\alpha+1) x^{2 p} \\
& +(p+2)(\alpha-2 p-1) x^{p+1} \\
& +p(2 p+1-\alpha) x^{p-1}-\alpha-1,
\end{aligned}
$$




$$
\lim _{t \rightarrow 1^{+}} h_{1}^{\prime}(x)=0,
$$

$h_{1}^{\prime \prime}(x)=x^{p-2} h_{2}(x)$,

where

$$
\begin{aligned}
h_{2}(x)= & 2(2 p+1)(p+1)(1-\alpha) x^{p+2} \\
& +2 p(2 p+1)(\alpha+1) x^{p+1} \\
& +(p+1)(p+2)(\alpha-2 p-1) x^{2} \\
& +(p-1) p(2 p+1-\alpha), \\
& \lim _{x \rightarrow 1^{+}} h_{2}(x)=0,
\end{aligned}
$$

$h_{2}^{\prime}(x)=2(p+1) x h_{3}(x)$,

where

$$
\begin{aligned}
& h_{3}(x)=(p+2)(2 p+1)(1-\alpha) x^{p} \\
&+p(2 p+1)(\alpha+1) x^{p-1} \\
&+(p+2)(\alpha-2 p-1), \\
& \lim _{x \rightarrow 1^{+}} h_{3}(x)=p(2 p-3 \alpha+1) \\
& h_{3}^{\prime \prime}(x)=p(2 p+1) x^{p-2} h_{4}(x)
\end{aligned}
$$

where

$$
\begin{gathered}
h_{4}(x)=(p+2)(1-\alpha) x+(p-1)(\alpha+1) \\
\lim _{x \rightarrow 1^{+}} h_{4}(x)=2 p-3 \alpha+1, \\
h_{4}^{\prime}(x)=(p+2)(1-\alpha) .
\end{gathered}
$$

We now distinguish between two cases.

Case 1. $p=\frac{3 \alpha-1}{2}$. We first consider the case $\alpha=\frac{1}{3}$ since in this case the one-parameter mean $J_{p}(a, b)$ has different expression from others. The result

$$
A^{\frac{1}{3}}(t, 1) G^{\frac{2}{3}}(t, 1)<J_{0}(t, 1)
$$

follows from Lemma 2.1 since

$$
A^{\frac{1}{3}}(t, 1) G^{\frac{2}{3}}(t, 1) / J_{0}(t, 1)=m^{3}(t, 1)<1,
$$

In the following we assume $\alpha \neq \frac{1}{3}$.

From (21) we see that $h_{4}^{\prime}(x)>0$ for $x>1$, which implies $h_{4}(x)$ is strictly increasing for $x>1$. From (20) we know that $h_{4}(x)>0$ for all $x>1$. (18) implies

$$
h_{3}^{\prime}(x) \begin{cases}<0, & \text { for } 0<\alpha<\frac{1}{3}, \\ >0, & \text { for } \frac{1}{3}<\alpha<1,\end{cases}
$$

from which we know $h_{3}(x)$ is strictly decreasing for $\alpha \in\left(0, \frac{1}{3}\right)$ and strictly increasing for $\alpha \in\left(\frac{1}{3}, 1\right)$. This result together with (17) implies $h_{3}(x)<0$ for $\alpha \in\left(0, \frac{1}{3}\right)$ and $h_{3}(x)>0$ for $\alpha \in\left(\frac{1}{3}, 1\right)$. The same reasoning applies to $h_{2}(x), h_{1}^{\prime \prime}(x), h_{1}^{\prime}(x), h_{1}(x)$ as well, and using (15), (14), (12), (11), (9) and (8), we know $g_{1}(t)<0 \quad$ for $\quad \alpha \in\left(0, \frac{1}{3}\right) \quad$ and $\quad g_{1}(t)>0 \quad$ for $\alpha \in\left(\frac{1}{3}, 1\right)$. (8) implies $f_{1}^{\prime}(t)>0$ for all $t>1$. Thus $f_{1}(t)$ is strictly increasing for $t>1$, which together with

$$
\lim _{t \rightarrow 1^{+}} f_{1}(t)=1
$$

implies right-hand side inequality of (8).

Case 2. $p=\frac{\alpha-1}{2}$. From (21) we know $h_{4}^{\prime}(x)>0$

for all $x>1$, which implies that $h_{4}(x)$ is strictly increasing for $x>1$. By (20) one has $h_{4}\left(1^{+}\right)=-2 \alpha<0$, and by (19) one has

$$
\lim _{x \rightarrow+\infty} h_{4}(x)=+\infty \text {. }
$$

Thus there exists $\xi_{1}>1$ such that $h_{4}(x)<0$ for $x \in\left(1, \xi_{1}\right)$ and $h_{4}(x)>0$ for $x \in\left(\xi_{1},+\infty\right)$. (18) implies $h_{3}^{\prime}(x)>0$ for $x \in\left(1, \xi_{1}\right)$ and $h_{3}^{\prime}(x)<0$ for $x \in\left(\xi_{1},+\infty\right)$. Thus $h_{3}(x)$ is strictly increasing for $x \in\left(1, \xi_{1}\right)$ and strictly decreasing for $x \in\left(\xi_{1},+\infty\right)$. By (17) $h_{3}\left(1^{+}\right)>0$ and by

$$
\lim _{x \rightarrow+\infty} h_{3}(x)=0
$$

we know $h_{3}(x)>0$ for all $x>1$. The same reasoning applies to $h_{2}^{\prime}(x), h_{2}(x), h_{1}^{\prime}(x), h_{1}(x)$ and $g_{1}(t)$ as well, and applying (9)-(16), we have $g_{1}(t)>0$ for all $t>1$. (9) implies $f_{1}^{\prime}(t)<0$, thus $f_{1}(t)$ is strictly decreasing for $t>1$. The left-hand side inequality of (8) follows from (22).

Next we prove that the bounds $J_{\frac{3 \alpha-1}{2}}(a, b)$ and $J_{\frac{\alpha-1}{2}}(a, b)$ are optimal.

For any $\varepsilon>0$ and $t>0$ sufficiently small,

$$
\begin{aligned}
& \log \frac{J_{\frac{3 \alpha-1}{2}-\varepsilon}(1+t, 1)}{A^{\alpha}(1+t, 1) G^{1-\alpha}(1+t, 1)} \\
& =\log \frac{4 t+(3 \alpha-2 \varepsilon-1) t^{2}}{4 t+(3 \alpha-2 \varepsilon-3) t^{2}}-\log \left(\frac{t+2}{2}\right)^{\alpha}(1+t)^{\frac{1-\alpha}{2}} \\
& =2 t^{2}-\alpha\left(\frac{t}{2}-\frac{t^{2}}{8}\right)-\frac{1-\alpha}{2}\left(t-\frac{t^{2}}{2}\right) \\
& =\frac{(18-\alpha) t-4}{8} t+o(t)<0 .
\end{aligned}
$$


This implies

$$
J_{\frac{3 \alpha-1}{2}-\varepsilon}(t, 1)<A^{\alpha}(t, 1) G^{1-\alpha}(t, 1)
$$

for $t$ sufficiently close to 1 .

For any $\varepsilon>0$, since

$$
\lim _{t \rightarrow+\infty} \frac{J_{\frac{\alpha-1}{2}+\varepsilon}(t, 1)}{A^{\alpha}(t, 1) G^{1-\alpha}(t, 1)}=+\infty,
$$

then there exists $T>1$ such that

$$
J_{\frac{\alpha-1}{2}+\varepsilon}(t, 1)>A^{\alpha}(t, 1) G^{1-\alpha}(t, 1)
$$

For $t>T$.

\section{Acknowledgements}

This research is supported by NSF of Hebei Province (No. A2011201011).

\section{REFERENCES}

[1] H. Y. Gao and W. J. Niu, "Sharp Inequalities Related to
One-Parameter Mean and Gini Mean,” Journal of Mathematical Inequalities, Vol. 6, No. 4, 2012, pp. 545-555. http://dx.doi.org/10.7153/jmi-06-52

[2] W. Cheung and F. Qi, "Logarithmic Convexity of the One-Parameter Mean Values,” Taiwanese Journal of Mathematics, Vol. 11, No. 1, 2007, pp. 231-237.

[3] M. K. Wang, Y. F. Qiu and Y. M. Chu, “An Optimal Double Inequality among the One-Parameter, Arithmetic and Harmonic Means," Revue d'Analyse Numerique et de Theorie de l'Approximation, Vol. 39, No. 2, 2012, pp. 169-175.

[4] H. N. Hu, G. Y. Tu and Y. M. Chu, "Optimal Bouds for the Seiffert Mean in Terms of One-Parameter Means," Journal of Applied Mathematics, 2012, Article ID: 917120.

[5] B. Y. Long and Y. M. Chu, "Optimal Inequalities for Generalized Logarithmic, Arithmetic and Geometric Mean," Journal of Inequalities and Applications, 2010, Article ID: 806825.

[6] N. G. Zheng, Z. H. Zhang and X. M. Zhang, "Schur-Convexity of Two Types of One-Parameter Mean Values in Variables,” Journal of Inequalities and Applications, 2007, Article ID: 78175. 